\title{
On Describing the Routing Capacity Regions of Networks
}

\author{
Ali Kakhbod*, S. M. Sadegh Tabatabaei Yazdi ${ }^{\dagger}$
}

November 21, 2018

\begin{abstract}
The routing capacity region of networks with multiple unicast sessions can be characterized using Farkas lemma as an infinite set of linear inequalities. In this paper this result is sharpened by exploiting properties of the solution satisfied by each ratetuple on the boundary of the capacity region, and a finite description of the routing capacity region which depends on network parameters is offered. For the special case of undirected ring networks additional results on the complexity of the description are provided.
\end{abstract}

Index Terms - routing, network capacity, multicast sessions, linear programming.

\section{Introduction}

Routing protocols underlie the traditional strategies for communicating information in data networks. The newer paradigm of network coding (see, e.g., Ahlswede et al. [2000]) offers potentially more reliable coding schemes with higher throughput and error correcting capabilities, but it is costlier to implement (see e.g., Langberg et al. [2006]). It is important to better understand routing because of its significance to the most practical networks. Furthermore, routing capacity regions provide inner bounds for the corresponding network coding capacity regions, and there are cases where the two capacity regions for the same networking problem are identical (e.g., Kramer and Savari [2006], Tabatabaei Yazdi et al. [2007b], Tabatabaei Yazdi et al. [2007a]).

We here focus on the routing capacity regions of a general class of networks supporting multiple multicast sessions. Much of the routing literature focuses on the multicommodity flow problem in which every message in the network is transmitted from a source to a unique destination (Stoenescu et al. [2007a], Stoenescu et al. [2007b]). The famous max-flow mincut theorem provides bounds on the rates of the different messages being simultaneously

\footnotetext{
${ }^{*}$ Electrical Engineering and Computer Science, University of Michigan, Ann Arbor, MI 48109. Email: akakhbod@umich.edu

${ }^{\dagger}$ Department of Electrical and Computer Engineering, Texas A\&M University, College Station, TX 77840. Email: sadegh@neo.tamu.edu
} 
transmitted between the different source-destination pairs. Schrijver [2003, Part VII] surveys many of the cases where this bound is tight. The paper $\mathrm{Hu}$ [1963] is an early reference which provides an example where the bound is not tight.

The papers Iri [1971] and Onaga [1970] establish a result sometimes called the "Japanese theorem", a special case of Farkas lemma, which provides necessary and sufficient conditions for determining if an arbitrary set of rates is feasible in a network. One shortcoming of this result is that the description of the routing capacity region for the multicommodity flow problem involves the intersection of an infinite set of inequalities. Another shortcoming of this result is that it only considers unicast sessions. While the assumption of a unique destination is natural for many application areas of network optimization, for communication problems we want to allow for the possibility of messages from a single transmitter to multiple receivers. Using standard terminology from communications, we further refer to unicast or multicast messages to indicate if the set of destinations is a single terminal or a set of multiple terminals. We will use the terms unicast and multicommodity flow interchangeably.

Just as one can form a system of linear inequalities to describe a multicommodity flow problem, one can likewise study the general multiple multicast problem where every terminal in the network potentially has messages for every non-empty subset of the other accessible terminals. For a multicommodity flow or unicast session the flow for a session which enters an intermediate vertex along the path is identical to the flow for that session emanating from that vertex. The natural generalization for multicast sessions constrains each spanning subtree carrying flow to have all of the edges of that subtree transmit the same flow. The set of flows along the various paths and subtrees are jointly constrained by the capacities of the edges or nodes in the graph, and the corresponding fractional routing capacity region can in principle be determined by Fourier-Motzkin elimination Schrijver [1998]. However, as the results of Fourier-Motzkin elimination are specific to the set of constraints for a particular networking problem, our objective is to offer a characterization which will apply to many networking problems.

The papers Tabatabaei Yazdi et al. 2007a] and Tabatabaei Yazdi et al. 2007b] extend the Japanese theorem to the networks with multiple multicast sessions. Roughly, they characterize the polytope of all achievable rates of a network with capacitated links in terms of an infinite set of inequalities, each corresponding to a distance function that assigns integer distances to the edges of the network. This characterization is valid for the multicast sessions, where the messages can have more than one destination. We will discuss this result and its proof in Section 2, and describe an inequality elimination technique to help study the network coding capacity region of special cases of the multiple multicast problem on an undirected ring network. The technique determines the minimal necessary and sufficient set of inequalities among the infinite set of inequalities specified by the Japanese theorem and 
is a consequence of properties of the routing solution for any rate-tuple on the boundary of the routing capacity region; this technique appears to be new even for the special case of multicommodity flow problems. We use it to further characterize the minimal set of inequalities for general directed or undirected networks and for undirected ring networks.

Our focus in this paper is on the size of the coefficients of the inequalities that appear in the minimal description of the routing rate region of an undirected network. We combine the inequality elimination technique with complexity results (see, e.g., Schrijver [1998], Grötschel et al. [1988]) on the description of a rational system of linear inequalities to bound the coefficients of the linear inequalities that describe the routing rate region. The obtained bounds are exponential in the number of edges of network. We further discuss an average case analysis of the size of linear inequalities for undirected ring networks, and by applying a probabilistic technique we suggest that for the characterization of routing capacity region we truly need to take into account the inequalities with the coefficients that grow polynomially in the number of edges of network.

The outline of the paper is as follows. In Section 2 we formulate and extend the Japanese theorem and describe an inequality elimination technique that was recently introduced in Tabatabaei Yazdi et al. [2007a] and Tabatabaei Yazdi et al. 2007b] to help study the routing capacity region. In Section 3 we present our results on the complexity of routing capacity regions of networks.

\section{The Routing Capacity Region of General Networks}

\subsection{Preliminaries}

Consider a network that is represented by a graph $G(V, E)$, where $V$ and $E$ respectively denote the set of vertices and edges in the network graph. The edges are either all undirected, meaning that the sum of flow along both directions of an edge is bounded by the capacity of the edge, or all directed. Furthermore, for any subgraph $S$ of the network let $V(S)$ and $E(S)$ respectively denote its set of vertices and edges. In a general communication setting, every vertex $v \in V$ can simultaneously send messages to arbitrary nonempty subsets of accessible vertices in $V \backslash\{v\}$. Every message $M$ with source node $v_{s}$ and set of destination nodes $\left\{v_{1}, \cdots, v_{k}\right\}$, is associated with a rate $R_{M}$ and with a set of $t(M)$ spanning subtrees, $\left\{T_{M}^{1}, \cdots, T_{M}^{t(M)}\right\}$, that connect $v_{s}$ to $\left\{v_{1}, \cdots, v_{k}\right\}$. We assume throughout that rates from any source to a set of vertices that include an inaccessible destination are always set to zero.

For message $M$, let $r_{M}^{j}$ be the amount of flow for that message that passes through 
spanning subtree $T_{M}^{j}, j \in\{1, \ldots, t(M)\}$. We then have

$$
\sum_{j=1}^{t(M)} r_{M}^{j}=R_{M} .
$$

Flows of the network satisfy the capacity constraints

$$
\sum_{M, j: e \in E\left(T_{M}^{j}\right)} r_{M}^{j} \leq C_{e}, e \in E,
$$

where $C_{e}$ denotes the capacity of edge $e$.

A rate-tuple $\mathcal{R}=\left(R_{M_{1}}, \cdots, R_{M_{N}}\right)$ corresponding to the sessions $M_{1}, \cdots, M_{N}$ is said to be feasible if for each $i \in\{1, \ldots, N\}$ there exists an assignment of $\left\{r_{M_{i}}^{1}, \cdots, r_{M_{i}}^{t\left(M_{i}\right)}\right\}$ such that

$$
\sum_{j=1}^{t\left(M_{i}\right)} r_{M_{i}}^{j}=R_{M_{i}}
$$

and the edge constraints are fulfilled. Our goal is to offer a new way of thinking about the set of feasible rate-tuples in a given network and to provide new characterizations of routing capacity regions.

\subsection{Generalizations of the Japanese Theorem}

The Japanese theorem characterizes the set of feasible routing rates-tuples for edge-constrained networks in problems where there are only multiple unicast sessions in terms of an infinite set of inequalities. Each inequality is in terms of a "distances function". A distance function is a function that assigns a positive integer to each edge in the network which is called the "distance" of the edge. For each inequality we further need to find the shortest path lengths for each session with respect to the corresponding distance function. The length of a path is the sum of distances of the edges on that path and the shortest path is the path with the shortest length. In what follows, $\mathrm{Z}^{+}$refers to the set of nonnegative integers.

Theorem 1 (Generalized Japanese theorem for edge-constrained networks Tabatabaei Yazdi et Consider a directed or an undirected edge-constrained network $G(V, E)$. For function $f$ : $E \rightarrow Z^{+}$, define $L_{f}(T)=\sum_{e \in E(T)} f(e)$ and $\ell_{f}(M)=\min _{j \in\{1, \ldots, t(M)\}} L_{f}\left(T_{M}^{j}\right)$. The rate-tuple $\mathcal{R}=\left(R_{M_{1}}, \cdots, R_{M_{N}}\right)$ is feasible in $G(V, E)$ if and only if for every function $f: E \rightarrow Z^{+}$, the following inequality holds:

$$
\sum_{i=1}^{N} \ell_{f}\left(M_{i}\right) R_{M_{i}} \leq \sum_{e \in E} f(e) C_{e} .
$$


To give an intuitive explanation of the Japanese theorem, consider a single unicast session in an edge constrained network. Then the famous max-flow min-cut theorem of Ford and Fulkerson provides the capacity of the transmission from the source node to the destination node. It is easy to verify that max-flow min-cut theorem is equivalent to considering the constraints of the form (1) when the distance functions are restricted to the functions with the range $\{0,1\}$ and each such function corresponds to an edge cut that separates the source node from the destination node. Each such function assigns value one to all edges that form a cut from the source node to the destination node and all other edges will be assigned a zero value. The Japanaese theorem states that in the case that all sessions are present in the network, the capacity of the network is characterized by all possible distance functions with the range of positive integers.

\subsection{An Inequality Elimination Technique}

Theorem 1 is unsatisfying because it describes the routing capacity region with infinitely many inequalities, and by Fourier-Motzkin elimination we know that the collection of feasible rate-tuples is a polytope defined by a finite set of inequalities. To circumvent this issue, we establish when a Japanese theorem inequality is redundant for a networking problem by exploiting the special structure of these inequalities. As we will see later, this approach enables us to offer a description of the set of feasible rate-tuples for the multiple multicast problem with only finitely many inequalities.

Every inequality in (1) is a description of a halfspace in the space of rate-tuples $\mathrm{R}^{N}$, and the feasible polytope of rate-tuples is the intersection of these half spaces with the half spaces corresponding to the non-negativity of rates. For the two types of network settings, the boundary points on the feasible set of rates respectively belong to the hyperplanes defined by:

$$
\begin{aligned}
& \sum_{i=1}^{N} \ell_{f}\left(M_{i}\right) R_{M_{i}}=\sum_{e \in E} f(e) C_{e}, \\
& \sum_{i=1}^{N} \ell_{f}\left(M_{i}\right) R_{M_{i}}=\sum_{v \in V} f(v) C_{v} .
\end{aligned}
$$

The following result provides the necessary and sufficient conditions for a feasible rate-tuple to be on a hyperplane of the form (2) or (3):

Theorem 2 Consider a directed network or an undirected network and fix any distance function $f$. The feasible rate tuple $\mathcal{R}=\left(R_{M_{1}}, \cdots, R_{M_{N}}\right)$ is on the hyperplane (2) or (3) if and only if there exists a feasible assignment $\left\{r_{M_{i}}^{j}: i \in\{1, \cdots, N\}, j \in\left\{1, \cdots, t\left(M_{i}\right)\right\}\right\}$ with the properties: 
1. $r_{M_{i}}^{j}=0$ if $L_{f}\left(T_{M_{i}}^{j}\right)>\ell_{f}\left(M_{i}\right)$, and for the appropriate setting

2. (Edge-constrained setting) $\sum_{\left\{i, j: e \in E\left(T_{M_{i}}^{j}\right)\right\}} r_{M_{i}}^{j}=C_{e}$ for $f(e)>0$

Proof: To establish the necessity of Conditions 1 and 2 in Theorem 2, suppose that the rate-tuple $\mathcal{R}=\left(R_{M_{1}}, \cdots, R_{M_{N}}\right)$ is feasible and is on the defining hyperplane (2) corresponding to function $f$. The sum of the flows passing through any edge $e$ in the network at most $C_{e}$. By multiplying both sides of this constraint by $f(e)$ and adding up the resulting inequalities over all edges in the network we find that

$$
\sum_{i=1}^{N} \sum_{j=1}^{t\left(M_{i}\right)} L_{f}\left(T_{M_{i}}^{j}\right) r_{M_{i}}^{j} \leq \sum_{e \in E} f(e) C_{e}
$$

A lower bound for the left-hand side of the preceding inequality is obtained when all sessions are routed along their shortest subtrees with respect to $f$ :

$$
\sum_{i=1}^{N} \ell_{f}\left(M_{i}\right) R_{M_{i}} \leq \sum_{i=1}^{N} \sum_{j=1}^{t\left(M_{i}\right)} L_{f}\left(T_{M_{i}}^{j}\right) r_{M_{i}}^{j} \leq \sum_{e \in E} f(e) C_{e} .
$$

Since the rate-tuple is on the hyperplane given by (2) by assumption, it follows that Condition 1 holds. To arrive at a contradiction, suppose next that Condition 2 is invalid. Hence the rate-tuple $\mathcal{R}$ is also feasible in another network with edge capacities $C_{e}^{\prime}, e \in E$, in which $C_{e}^{\prime} \leq C_{e}$ for all $e$ with strict inequality for at least one value of $e$ with $f(e)>0$. Then Theorem 1 implies that

$$
\sum_{i=1}^{N} \ell_{f}\left(M_{i}\right) R_{M_{i}} \leq \sum_{e \in E} f(e) C_{e}^{\prime}<\sum_{e \in E} f(e) C_{e}
$$

which contradicts (2). Thus Condition 2 holds.

To establish sufficiency, consider a feasible rate-tuple which satisfies Conditions 1 and 2. The argument for constraint (4) applies for any feasible point, and Condition 2 implies that (41) can be replaced by the equality $\sum_{i=1}^{N} \sum_{j=1}^{t\left(M_{i}\right)} L_{f}\left(T_{M_{i}}^{j}\right) r_{M_{i}}^{j}=\sum_{e \in E} f(e) C_{e}$. By Condition 1 we further know that

$$
\sum_{i=1}^{N} \ell_{f}\left(M_{i}\right) R_{M_{i}}=\sum_{i=1}^{N} \sum_{j=1}^{t\left(M_{i}\right)} L_{f}\left(T_{M_{i}}^{j}\right) r_{M_{i}}^{j}=\sum_{e \in E} f(e) C_{e} .
$$

Hence, the rate-tuple $\mathcal{R}$ is on the defining hyperplane corresponding to function $f$, completing the proof.

We focus here on distance functions $f$ that result in nontrivial inequalities with $\ell_{f}\left(M_{i}\right)>$ 0 for at least one $i \in\{1, \ldots, N\}$. We say that a nontrivial Japanese theorem inequality is 
redundant if for any assignment of capacities the feasible rate-tuples on the corresponding defining hyperplane all lie on the hyperplane bounding another nontrivial Japanese theorem inequality. We will next demonstrate that Theorem 2 implies a way to establish whether or not an inequality coming from the Japanese theorem or its extensions is redundant for a given networking problem. In the next section we will show that our inequality elimination technique enables us to characterize the fractional routing capacity region for the multiple multicast problem with a finite number of inequalities. As we will see, the true significance of the distance function is summarized by the collections of shortest paths for the unicast sessions and shortest subtrees for the multicast sessions corresponding to that function.

Theorem 3 (Elimination Theorem) Given an edge-constrained network with a set of messages $\left\{M_{1}, \cdots, M_{N}\right\}$, consider two nontrivial distance functions $f$ and $g$ which are not identical. The network may be either directed or undirected. If

1. for every $e \in E, f(e)=0$ whenever $g(e)=0$, and

2. for every session $M_{i}, i \in\{1, \cdots, N\}$ and for all $j \in\left\{1, \cdots, t\left(M_{i}\right)\right\}$ the property $L_{g}\left(T_{M_{i}}^{j}\right)=\ell_{g}\left(M_{i}\right)$ implies $L_{f}\left(T_{M_{i}}^{j}\right)=\ell_{f}\left(M_{i}\right)$ (but not necessarily the converse),

then the half space (1) corresponding to $g$ is redundant in the description of the fractional routing capacity region given the half space corresponding to $f$.

Before we prove this result, we will illustrate it with an example. Consider an undirected triangle network with $V=\{1,2,3\}$ and suppose $C_{(1,2)}=C_{(2,3)}=C_{(3,1)}=1$. We permit all possible unicast and multicast sessions. Take $g((1,2))=2, g((2,3))=1$, and $g((3,1))=3$. It is easy to verify

- $\ell_{g}(1 \rightarrow 2)=\ell_{g}(2 \rightarrow 1)=2$ and the shortest path is $(1,2)$,

- $\ell_{g}(2 \rightarrow 3)=\ell_{g}(3 \rightarrow 2)=1$ and the shortest path is $(2,3)$,

- $\ell_{g}(3 \rightarrow 1)=\ell_{g}(1 \rightarrow 3)=3$ and both paths are shortest, and

- $\ell_{g}(1 \rightarrow\{2,3\})=\ell_{g}(2 \rightarrow\{1,3\})=\ell_{g}(3 \rightarrow\{1,2\})=3$ and the shortest subtree is $\{(1,2),(2,3)\}$.

Therefore, the half space corresponding to distance function $g$ is

$$
\begin{gathered}
2\left(R_{1 \rightarrow 2}+R_{2 \rightarrow 1}\right)+\left(R_{2 \rightarrow 3}+R_{3 \rightarrow 2}\right)+3\left(R_{3 \rightarrow 1}+R_{1 \rightarrow 3}\right)+3\left(R_{1 \rightarrow\{2,3\}}+R_{2 \rightarrow\{1,3\}}+R_{3 \rightarrow\{1,2\}}\right) \\
\leq 2 C_{(1,2)}+C_{(2,3)}+3 C_{(3,1)}=6 .
\end{gathered}
$$

Next take $f((1,2))=1, f((2,3))=0$, and $f((3,1))=1$. Notice that the shortest paths and shortest subtrees for each session under distance function $g$ remain shortest paths and 
shortest subtrees for the sessions under $f$, although $f$ has a second shortest path for unicast sessions $1 \rightarrow 2$ and $2 \rightarrow 1$ and a second shortest subtree for the multicast sessions. The halfspace corresponding to $f$ is

$$
\left(R_{1 \rightarrow 2}+R_{2 \rightarrow 1}\right)+\left(R_{3 \rightarrow 1}+R_{1 \rightarrow 3}\right)+\left(R_{1 \rightarrow\{2,3\}}+R_{2 \rightarrow\{1,3\}}+R_{3 \rightarrow\{1,2\}}\right) \leq C_{(1,2)}+C_{(3,1)}=2 .
$$

The theorem states that (8) is redundant for defining the routing capacity region in the presence of (9). The reason is that a polytope is defined by a collection of hyperplanes, and every feasible rate-tuple like $R_{1 \rightarrow 2}=R_{2 \rightarrow 3}=R_{3 \rightarrow 1}=1, R_{M}=0, M \notin\{1 \rightarrow 2,2 \rightarrow$ $3,3 \rightarrow 1$ \} which satisfies (8) with equality must also satisfy (9) with equality. The ratetuple $R_{1 \rightarrow\{2,3\}}=2, R_{M}=0, M \notin\{1 \rightarrow\{2,3\}\}$ is an example of an infeasible rate-tuple which satisfies (8) with equality; it is infeasible because four units of capacity are needed to transmit two units of multicast traffic, and the network has only three units of capacity. For the problem of characterizing the routing capacity region of a network we can ignore the infeasible rate-tuples.

We assert here that there are generalizations of Theorem 3 to some other classes of systems of linear inequalities. To save space we only give the proof for the edge-constrained setting from Tabatabaei Yazdi et al. [2007b]. Tabatabaei Yazdi et al. [2007a] offers an alternate proof of the same result which uses the formalism of linear algebra.

Proof: Consider a feasible rate-tuple on the hyperplane (1) corresponding to $g$. By Condition 1 of Theorem 2, every session is routed only along the shortest paths and shortest subtrees associated with $g$, and hence by assumption only along the shortest paths and shortest subtrees corresponding to $f$. Furthermore, note that by assumption any edge $e$ with $f(e)>0$ implies $g(e)>0$ and so Condition 2 of Theorem 2 implies that this edge must be fully utilized. By Theorem 2 it follows that the feasible rate-tuple is also on the hyperplane (11) corresponding to $f$. Since the routing capacity region can be described in terms of its defining hyperplanes, the bound corresponding to $g$ is redundant given the inequality corresponding to $f$.

The paper Tabatabaei Yazdi et al. [2007b] considers undirected, edge-constrained ring networks with multiple unicast and broadcast sessions; in a broadcast session the source transmits a message to all of the other vertices in the network. That paper introduced the inequality elimination technique and used it to prove that distance functions with range $\{0,1\}$ are sufficient for characterizing the capacity region. The paper Tabatabaei Yazdi et al. [2007a] proved that $\{0,1\}$ edge distances are also enough to describe the multiple multicast capacity region of undirected, edge-constrained ring networks in which the source and destination vertices of each communication session form a string of adjacent vertices. We next present new consequences of the inequality elimination theorem. 


\section{On the Complexity of the Routing Capacity Region}

We next consider the complexity of the routing capacity region for an undirected graph. Let $p$ and $q$ be relatively prime integers and let $\alpha=p / q$. Define

$$
\operatorname{size}(\alpha)=1+\left\lceil\log _{2}(1+|p|)\right\rceil+\left\lceil\log _{2}(1+|p|)\right\rceil \text {. }
$$

For the rational vector $\mathbf{c}=\left(\gamma_{1}, \cdots, \gamma_{n}\right)$ and the rational matrix $A=\left(\alpha_{i, j}\right)_{1 \leq i \leq m, 1 \leq j \leq n}$ we have:

$$
\begin{array}{r}
\operatorname{size}(\mathbf{c})=n+\operatorname{size}\left(\gamma_{1}\right)+\cdots+\operatorname{size}\left(\gamma_{n}\right) \\
\operatorname{size}(A)=m n+\sum_{m, n} \operatorname{size}\left(\alpha_{i, j}\right)
\end{array}
$$

Let $\mathbf{x}=\left(x_{1}, x_{2}, \cdots, x_{n}\right)^{T}$. Then the size of the linear inequality $\mathbf{a x} \leq \alpha$ is defined as $1+\operatorname{size}(\mathbf{a})+\operatorname{size}(\alpha)$. The size of a system $A \mathbf{x} \leq \mathbf{b}$ of linear inequalities is defined as $1+\operatorname{size}(A)+\operatorname{size}(\mathbf{b})$. Next let $P \subset \mathcal{R}^{n}$ be a rational polyhedron. The facet complexity of $P$ defined as the smallest number $\Lambda \geq n$ for which there exists a system $A \mathbf{x} \leq \mathbf{b}$ of rational linear inequalities defining $P$ and each inequality in $A \mathbf{x} \leq \mathbf{b}$ has size at most $\Lambda$.

Consider an undirected network $G(V, E)$ and the rate-tuple $\mathcal{R}=\left(R_{M_{1}}, \cdots, R_{M_{N}}\right)$. Let $P$ denote the set of achievable rate-tuples in $\mathbb{R}^{N}$. Theorem 3 provides a systematic method to characterize the minimal description of $P$ for the general multiple multicast problem. Here we wish to establish upper and lower bounds on the maximum values of the functions that appear in the minimal description of $P$.

In the case of an undirected ring network with $|E|$ edges, Tabatabaei Yazdi et al. 2007b] points out that for the general multiple multicast problem there is a weight vector with maximum edge distance $\lfloor(|E|-1) / 2\rfloor$ that cannot be eliminated.

We next show that for undirected ring networks we have to consider some distance functions where the maximum edge distance grows at least exponentially in $|E|$. In words, we derive a lower bound on the maximum values of the distance functions needed for the minimum description of the corresponding routing rate region, which is exponential in $|E|$. For that matter, we construct a distance function $g$ that can not be eliminated by any nontrivial distance function with the maximum edge distance less than $2^{\lfloor(|E|-2) / 3\rfloor}$. The distance function $g$ is not unique and it is constructed as to achieve a bound in the same order of the upper bound obtained in Theorem 6 . 
Theorem 4 Let $G(V, E)$ be an undirected ring network with vertices $1,2, \cdots,|E|$ in a clockwise direction. For $i \in\{1,2, \ldots,|E|-1\}$, let edge $i$ connect vertices $i$ and $i+1$, and let edge $|E|$ connect vertices $|E|$ and 1 . There exist a distance function $g$ that cannot be eliminated by any nontrivial distance function $f$ with $\max _{e \in E} f(e)<2^{\lfloor(|E|-2) / 3\rfloor}$.

Proof: Consider a multicast session with $k-1$ destinations, and suppose the source and destination vertices form the set $\left\{v_{1}, v_{2}, \ldots, v_{k}\right\}$, where $1 \leq v_{1}<v_{2}<\cdots<$ $v_{k} \leq|E|$. Observe that a minimal spanning subtree is the subgraph consisting of the original network except for the vertices $v_{j}+1, \ldots, v_{j+1}-1$ and edges $v_{j}, \ldots, v_{j+1}-1$ for some $j \in\{1, \ldots, k\}$ (with $v_{k+1}=v_{1}$ ). Therefore, for any distance function the shortest paths or shortest subtrees for this collection of sessions will correspond to the longest paths $v_{j}, \ldots, v_{j+1}-1, j \in\{1, \ldots, k\}$.

Let $\beta=2^{\lfloor(|E|-2) / 3\rfloor}$. Suppose we consider the distance function

$$
g(e)= \begin{cases}\beta, & e \equiv 1 \quad(\bmod 3) \\ 2^{\lfloor(e-2) / 3\rfloor}, & \text { otherwise }\end{cases}
$$

and try to find another distance function $f$ that eliminates $g$. Since the shortest broadcast trees are preserved under $f$, it follows that

$$
\max _{i \in E} f(i)=f(e), e \equiv 1 \quad(\bmod 3)
$$

Furthermore, for $s \in\{2, \ldots,\lfloor|E| / 3\rfloor\}$ consider the multicast session consisting of all vertices except $3 s-4,3 s-3$, and $3 s-1$. Under $g$, the path consisting of edges $3 s-5,3 s-$ $4,3 s-3$, and the path consisting of edges $3 s-2$ and $3 s-1$ are both longest, and therefore (12) implies

$$
f(3 s-4)+f(3 s-3)=f(3 s-1), s \in\{2, \ldots,\lfloor|E| / 3\rfloor\} .
$$

Finally, for $s \in\{2, \ldots,\lfloor(|E|+1) / 3\rfloor\}$ consider the multicast session consisting of all vertices except $3 s-4$ and $3 s-2$. Under $g$, the path consisting of edges $3 s-5$ and $3 s-4$ and the path consisting of edges $3 s-3$ and $3 s-2$ are both longest, and therefore (12) implies

$$
f(3 s-4)=f(3 s-3), s \in\{2, \ldots,\lfloor(|E|+1) / 3\rfloor\} .
$$

By (13) and (14), we see that

$$
2 f(3 s-4)=f(3 s-1), s \in\{2, \ldots,\lfloor|E| / 3\rfloor\} .
$$

Equations (12)-(15) imply that $f(e)=f(2) \cdot g(e)$ for all $e \in E$.

Let $\Lambda^{*}$ denote the maximum distance among distance functions used for a shortest description of $P$. Theorem 4 establishes that $\Lambda^{*} \geq 2^{\lfloor(|E|-2) / 3\rfloor}$. We next extend Theorem 4 
to any undirected graph.

Corollary 5 Given undirected graph $G(V, E)$ with maximum cycle length $m$, for the networking problem in which all possible multicast sessions are supported, the minimum description of the corresponding routing rate region requires a distance function with $\Lambda^{*} \geq$ $2^{\lfloor(|E|-2) / 3\rfloor .}$

Proof: Let $\mathcal{C}$ denote a maximum cycle of $G$. We extend the proof of Theorem 4 by using the distance function $f$ along $\mathcal{C}$ and setting $f(e), e \notin \mathcal{C}$, to be sufficiently large.

Next we bound $\Lambda^{*}$ from above.

Theorem 6 For an undirected or a directed network $G(V, E), \Lambda^{*} \leq 2^{24|E|^{3}+8|E|^{2}}$.

Proof: Suppose that the distance vector $\mathbf{f}=(f(1), \cdots, f(|E|))$ belongs to the minimal description of $P$. We form the homogeneous set of inequalities $A \mathrm{~g} \leq 0$ such that $\mathbf{g}: A \mathbf{g} \leq 0, \mathbf{g} \in \mathbb{Z}^{|E|}$ is the set of all distance vectors that can eliminate $\mathbf{f}$ by the criteria given in Theorem 3. This includes all inequalities that describe the shortest subtrees for every session corresponding to function $f$, and also the non-negativity of elements of g. Notice that this set is non-empty since $\mathbf{f}$ is a solution to it. Furthermore, all elements of matrix $A$ are in $\{0,+1,-1\}$. This implies the upper bound $3|E|+1$ on the size of the inequalities in $A \mathrm{~g} \leq 0$. Therefore the facet complexity of $A \mathbf{g} \leq 0$, is at most $\Lambda_{A}=3|E|+1$. [Schrijver [1998], Theorem 10.2] implies that $A \mathbf{g} \leq 0$ has a rational solution of size at most $4|E|^{2} \Lambda_{A}=12|E|^{3}+4|E|^{2}$. Let $\mathbf{g}_{r}=\left(p_{1} / q_{1}, \cdots, p_{|E|} / q_{|E|}\right)$ denote such a solution. Since $A \mathrm{~g} \leq 0$ is a homogeneous set of inequalities, any integral multiple of $\mathbf{g}_{r}$ is also a solution to $A \mathbf{g} \leq 0$. Now consider the vector $\mathbf{g}_{z}=\left(q_{1} \cdots q_{|E|}\right) \mathbf{g}_{r}$. Clearly $\mathbf{g}_{z} \in\left\{\mathbf{g}: A \mathbf{g} \leq 0, \mathbf{g} \in \mathbb{Z}^{|E|}\right\}$, so it can eliminate $\mathbf{f}$. Let $\mathbf{g}_{z}(i)$ be the maximum entry of $\mathbf{g}_{z}$. Then size $\left(\mathbf{g}_{z}(i)\right) \leq \operatorname{size}\left(q_{1} \cdots q_{|E|}\right)+\operatorname{size}\left(\mathbf{g}_{r}(i)\right)$. Since size $\left(q_{1} \cdots q_{|E|}\right) \leq \operatorname{size}\left(\mathbf{g}_{r}\right)$ and $\operatorname{size}\left(\mathbf{g}_{r}(i)\right) \leq \operatorname{size}\left(\mathbf{g}_{r}\right)$, then size $\left(\mathbf{g}_{z}(i)\right) \leq 24|E|^{3}+8|E|^{2}$. This yields the result.

The following result, suggests that a small fraction of the distance functions in our characterization of the fractional routing capacity region are truly needed and that most distance functions can be eliminated by distance functions where the maximum entry grows polynomially with $|E|$.

Theorem 7 Let $G(V, E)$ be an undirected ring network with edges labeled 1, 2, $\cdots,|E|$ in a clockwise order. Choose any integer $m \geq 6$, and suppose $g_{\max }=\max _{e \in E} g(e)>g^{*} \doteq$ $|E|^{m} /\left(1-\frac{|E|^{m}}{g_{\max }}\right)$. Assume without loss of generality that $g(|E|)=g_{\max }$ and for $e \in E \backslash|E|$ let $g(e)$ be uniformly chosen among nonnegative integers less than or equal to $g_{\max }$. Then with probability at least $1-\frac{4}{|E|^{m-5}}-\frac{1}{|E|^{m(|E|-1)}}$ we can find a distance function $f$ with $f_{\max } \leq g^{*}$ that eliminates $g$. 
Proof: Given distance function $g$ with $g_{\max } \geq g^{*}$, let $\phi=\left\lfloor g_{\max } /|E|^{m}\right\rfloor$, and define

$$
f(e)=g(e)-(g(e) \quad(\bmod \phi)), e \in E .
$$

Distance function $f$ eliminates distance function $g$ if for any pair of edge-disjoint subtrees $E_{1}$ and $E_{2}$ of $E$, the condition $\sum_{e \in E_{1}} g(e) \leq \sum_{i \in E_{2}} g(e)$ implies $\sum_{e \in E_{1}} f(e) \leq \sum_{i \in E_{2}} f(e)$. Let $\mathcal{E}_{E_{1}, E_{2}}$ be the event that $\sum_{e \in E_{1}} g(e) \leq \sum_{e \in E_{2}} g(e)$ and $\sum_{e \in E_{1}} f(e)>\sum_{e \in E_{2}} f(e)$. Define

$$
\begin{aligned}
\Delta_{g} & =\sum_{e \in E_{1}} g(e)-\sum_{e \in E_{2}} g(e) \\
\text { and } \Delta_{f} & =\sum_{e \in E_{1}} f(e)-\sum_{i \in E_{2}} f(e) .
\end{aligned}
$$

Since $0 \leq g(e)-f(e)<\phi$ for all $e \in E$, it follows that

$$
\left|\Delta_{g}-\Delta_{f}\right| \leq \sum_{e \in E}|g(e)-f(e)|<\phi \cdot|E|
$$

We know that $\Delta_{g} \leq 0$ and $\Delta_{f}>0$, and so $\left|\Delta_{g}\right|<\phi \cdot|E|$. Let $E_{\min }=\min _{e \in E_{1} \cup E_{2}} e$. Observe that $E_{\min } \neq|E|$. Given $g(e), \leq e \in E \backslash E_{\min }$, there are at most $2 \phi \cdot|E|$ choices for $g\left(E_{\min }\right)$ that result in $-\phi \cdot|E|<\Delta_{g} \leq 0$. Furthermore $g\left(E_{\min }\right)$ is a random variable uniformly distributed over the integers between 0 and $g_{\max }$. Therefore,

$$
\mathbb{P}\left(\mathcal{E}_{E_{1}, E_{2}}\right) \leq \frac{2 \phi \cdot|E|}{g_{\max }+1}<\frac{2 \cdot \frac{g_{\max }}{|E|^{m}} \cdot|E|}{g_{\max }}=\frac{2}{|E|^{m-1}} .
$$

The number of pairs of edge-disjoint subtrees $E_{1}$ and $E_{2}$ we need consider is less than $2|E|^{4}$. Hence,

$$
\mathbb{P}\left(\bigcup_{E_{1}, E_{2}} \mathcal{E}_{E_{1}, E_{2}}\right)<2|E|^{4} \cdot \frac{2}{|E|^{m-1}}=\frac{4}{|E|^{m-5}} .
$$

In addition, In order to omit the trivial cases, a distance function is not trivial if and only if there exist at least $e$ and $e^{\prime}$ in $E$ such that $f(e)>0$ and $f\left(e^{\prime}\right)>0$, since for all $e \in E$, $\mathbb{P}(f(e)=0)<\frac{1}{|E|^{m}}$, Thus, with probability at least $1-\frac{4}{|E|^{m-5}}-\frac{1}{|E|^{m(|E|-1)}}$ we can use distance function $f$ to eliminate $g$. Since $f(e)(\bmod \phi)=0$ for all $e \in E$, we can eliminate distance function $f$ by distance function $f^{*}$ with $f^{*}(e)=f(e) / \phi, e \in E$. Notice that for all $e \in E$,

$$
\begin{gathered}
f^{*}(e)=\frac{f(e)}{\phi} \leq \frac{g(e)}{\phi} \leq \frac{g_{\max }}{\left[g_{\max } /|E|^{m}\right\rfloor}<\frac{g_{\max }}{\frac{g \max }{|E|^{m}}-1} \\
=\frac{|E|^{m}}{1-\frac{|E|^{m}}{g_{\max }}} .
\end{gathered}
$$




\section{Acknowledgements}

The authors are gratefully thankful to Serap Savari for her insightful and encouraging discussions on the material of this paper.

\section{References}

R. Ahlswede, N. Cai, S. R. Li, and R. W. Yeung. Network information flow. IEEE Transactions on Information Theory, 46:1204-1216, 2000.

M. Grötschel, L. Lovász, and A. Schrijver. Geometric Algorithms and Combinatorial Optimization, volume 2 of Algorithms and Combinatorics. Springer, 1988. ISBN 3-540-13624X, 0-387-13624-X (U.S.).

T. C. Hu. Multi-commodity network flows. Operations Research, 11:344-360, 1963.

M. Iri. On an extension of the maximum-flow minimum-cut theorem to multicommodity flows. J. Operations Research Soc. of Japan, 13:129-135, 1971.

G. Kramer and S. A. Savari. Edge-cut bounds on network coding rates. J. Network Sys. Management, 14:49-67, 2006.

M. Langberg, A. Sprintson, and J. Bruck. The encoding complexity of network coding. in Proc. IEEE ISIT 05, 52:1987-1991, 2006.

K. Onaga. A multi-commodity flow theorem. Electronics and Communications in Japan, 53-A:7:16-22, 1970.

A. Schrijver. Theory of Linear and Integer Programming. New York: Wiley, 1998.

A. Schrijver. Combinatorial Optimization. New York: Springer, 2003.

T. Stoenescu, M. Liu, and D. Teneketzis. Multi-rate multicast service provisioning, part i: an algorithm for optimal price splitting along multicast trees. Mathematical Methods of Operations Research, 65:199-228, 2007a.

T. Stoenescu, M. Liu, and D. Teneketzis. Multi-rate multicast service provisioning, part ii: a tatonnement process for rate allocation. Mathematical Methods of Operations Research, 65:389-415, 2007b. 
S. M. Tabatabaei Yazdi, S. A. Savari, K. Carlson (Talaska), and G. Kramer. The capacity region of a collection of multicast sessions in undirected ring network. In Proc. Int. Workshop on Network Coding (IWNC) as part of the 36th Int. Conf. Parallel Proc. (ICPP), Xian, China, 2007a.

S. M. Tabatabaei Yazdi, S. A. Savari, F. Farnoud, and G. Kramer. A multimessage capacity region for undirected ring networks. In Proc. 2007 IEEE Int. Symp. Inform. Theory, pages 1091-1095, Nice, France, 2007b. 